\title{
Characterizing the Quiet Sun Scale Magnetic Field
}

\author{
Alan Title ${ }^{1}$ \\ ${ }^{1}$ Lockheed Martin Advanced Technology Center, 3251 Hanover Street, Palo Alto, 94304, USA
}

\begin{abstract}
Observations with the Solar Optical Telescope on Hinode indicate that the Quiet Sun magnetic field occurs on every scale of convection including granulation. Data reported here show that, regardless of the position on the disk, the polarity in the inner network regions are balanced to 1 part in 72 . This is consistent with both local dynamo processes or the creation of surface features by the granulation downflows.
\end{abstract}

Keywords. Sun: magnetic fields

\section{Introduction}

For a number of years there has been evidence of the presence of magnetic fields virtually everywhere on the surface of the Quiet Sun (QS). This has suggested that flux was appearing at the surface on the scale of granulation in a manner similar to the now well established "magnetic carpet" which maintains the kilogauss field elements observed in the supergranule boundaries. MDI data had shown that the carpet is sustained by continuous emergence of "ephemeral regions", magnetic structures with an average flux a few times 1018 Mx. High sensitivity magnetograph and spectro-polarimeter (SP) measurements have long shown the existence of "internet-work fields" structures with fluxes as low as $1015 \mathrm{Mx}$. While there is general agreement that the network and ephemeral fields have strengths on the order of a kilogauss, there has remained some controversy about the strength of the internet-work fields. Their estimated strengths have ranged between a few hundred gauss and a kilogauss.

Careful measurements of MDI time sequences have revealed the presence and rapid evolution of "magnetic fragments". Fragments are different from ephemeral regions in that they are not easily associated with an opposite polarity mate or mates. The number of fragments increases with increased spatial resolution of magnetic maps. This has led to the statement that more flux seems to appear on the Sun in "unassociated" fragments than recognizable bipoles. The abundance of fragments can not indicate that there are magnetic monopoles, but rather that the emergence of flux on the surface can take complex and not easily recognized forms. Numerical simulations of flux emergence and local dynamo action exhibit patterns of appearance in which bipole emergence would be impossible to recognize at the resolution of current telescopes.

Many new insights into the characteristics of the QS magnetic field have arisen from the observations made with the Solar Optical Telescope (SOT) on the Hinode. These insights have their origin in the uniform image quality allowed from the seeing-free space platform; the lack of the need for extensive image processing techniques to increase resolution that tend to diminish or eliminate low contrast transient features; the combination of a very sensitive SP and a filter magnetograph; and a set of powerful and efficient software tools for construction and analysis of movies and SP maps.

The first observations made with both the birefringent filter magnetograph and SP revealed magnetic field virtually everywhere in the QS. High signal-to-noise spectra showed 
that granule boundaries were nearly everywhere filled with mixed polarity near vertical magnetic fields, while horizontal fields spread over much of the entire surface. The presence of a pervasive sea of flux on the granular scale has immediate implications because the granular pattern has a lifetime on the order of ten minutes. A consequence of the granular flows is that flux is continuously swept into the granule boundaries on the granule turn over time. In order to always observe flux on the spatial scale of granulation, flux must be continuously emerging on the granule temporal scale. Figure 1 shows a continuum image and a map of the vertical field in a region of very QS. Magnetogram contours overlaid on the continuum image illustrate that the majority of the magnetic flux is in the granule boundaries. Nevertheless, there are also clear examples of magnetic signal in granules. This should be expected both in the case that all flux does not emerge in boundaries or when during the initial stages of emergence there is a significant vertical component over the center of granules.
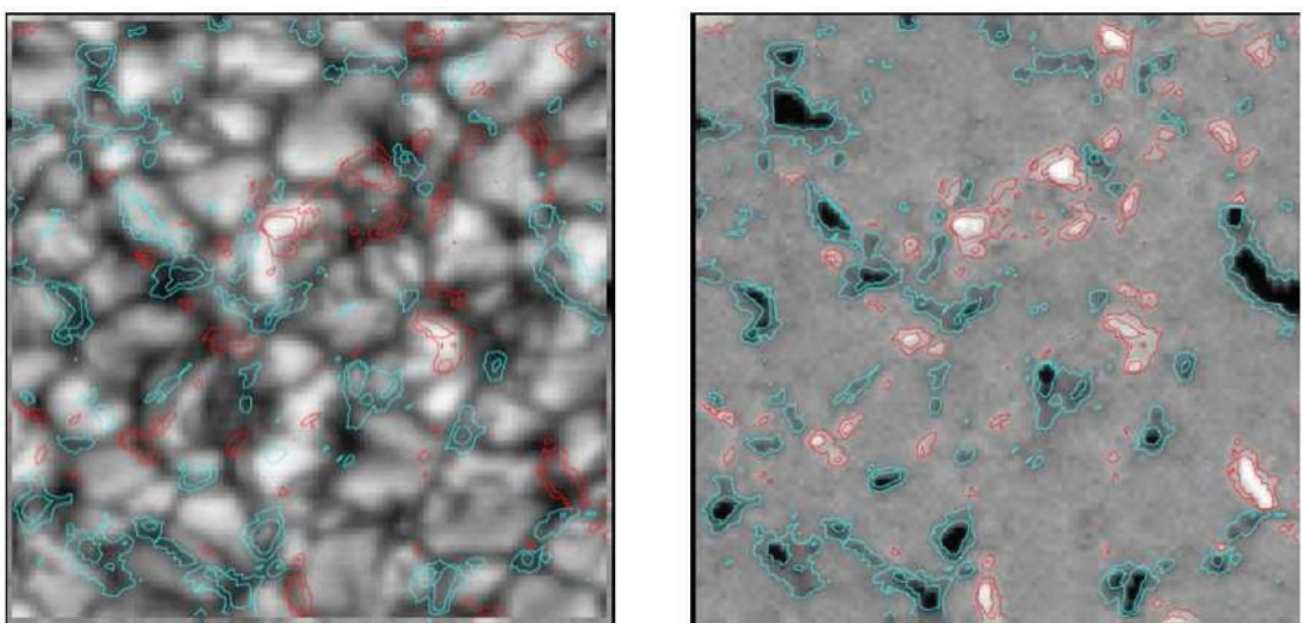

Figure 1. Continuum image (left) overlaid by the contours of the vertical magnetic field (right). The image and the magnetic map were constructed from a SP scan.

Hinode filter magnetograph movies show that active regions emerge in a very complex manner. Between the flux concentrations that eventually contract into the leading and trailing parts of the active region there emerges a sea of smaller bipoles roughly aligned with the two growing regions of opposite polarity (see figure 2). This has been seen previously and even semi-empirical models had suggested that a phenomena, O-loops, could explain the observation that sunspots seem to grow nearly in place. What the new Hinode observations have shown is that many of the same features seen in emergence of active regions are repeated in emergence events that are much smaller (see figure 3 ). The Hinode data suggests that much of the flux that appears on the solar surface does not initially emerge as a single bipole, but as a sea of small flux structures each of which is oriented in roughly the same direction. The smaller the total flux in an emergence event the more random the orientation of the components and the shorter their lifetime on the solar surface. Because of the complexity of the emergence patterns it is difficult to impossible to associate a particular flux element with the opposite polarity feature or features with which it emerged. In part this is caused by merging that has occurred 
before the flux elements have reached the surface. Given the Hinode observations, it is not surprising that more fragments have been observed than bipole pairs in earlier data.

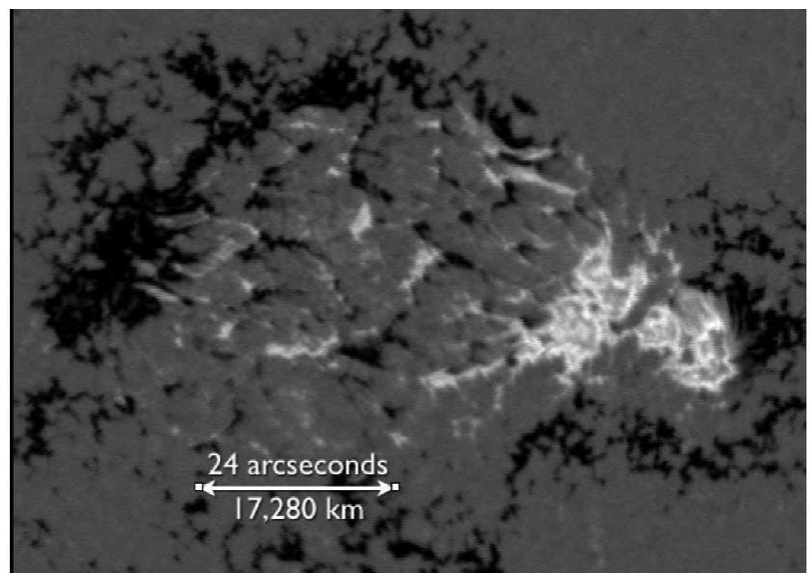

Figure 2. An evolving active region on 11 December 2007 showing the emergence of intermediate bipoles between the leading and following flux concentrations.

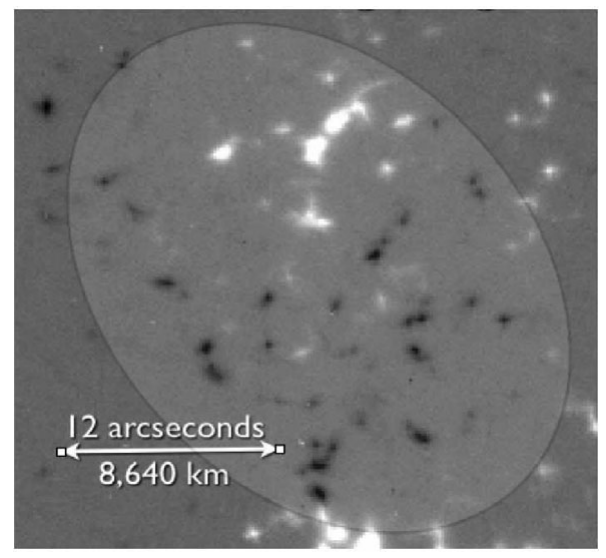

Figure 3. An example of a complex emergence of an array of small magnetic flux elements. The magnetogram is from a frame of a movie of QS on 5 November 2007. It is difficult to identify bipoles in this complex pattern either in this single frame or in the movie.

Numerical models of how a flux tube started $5000 \mathrm{~km}$ below the visible surface emerges exhibit features that have a remarkable similarity to the Hinode observations described above. The rapid decrease in the atmospheric pressure near the surface requires that the magnetic pressure in the tube decreases in order to maintain hydrostatic equilibrium. As a result a uniform circular flux tube that started $5000 \mathrm{~km}$ below the surface expands perpendicular to its axis as it rises forming a relatively thin flat oval of horizontal flux just below the surface. Examples of the start and the evolution of such a numerical simulation is shown in figure 4. As the "pond" of flux forms near the surface, strong granular downflows drag the magnetic field lines downward creating local bipoles. Because the pond can have dimensions larger than the spacing of the convective downflows, the total 
(unsigned) flux that emerges on the surface can be greater than the total flux in a cross section of the original emerging flux tube. In figure 5 a pair of $3 \mathrm{D}$ slices show the flux pattern at $5000 \mathrm{~km}$ and just below below the surface. A few field lines are shown that illustrate the formation of bipoles that are created by the convective downflows.

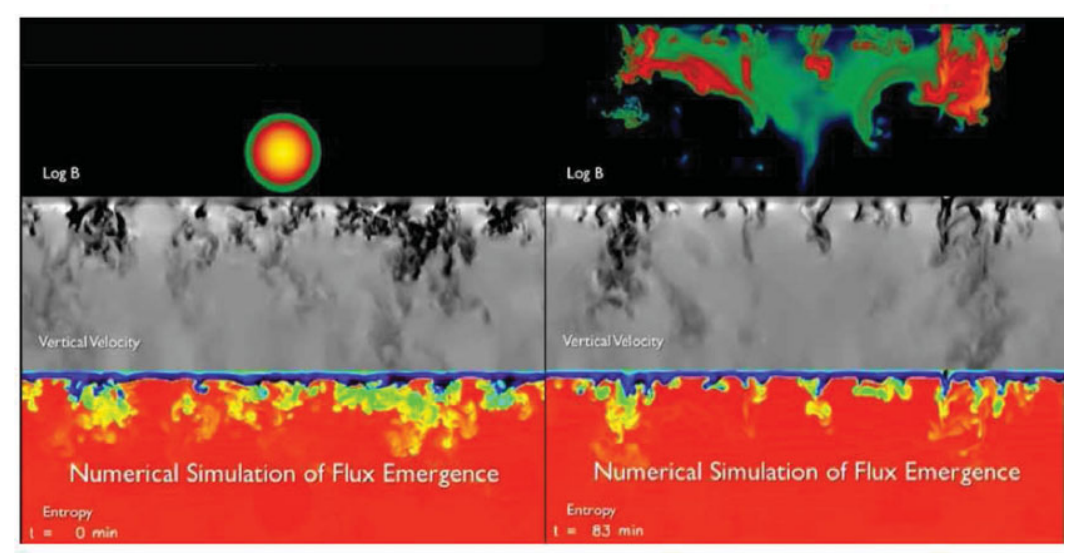

Figure 4. Cross section of a rising originally circular flux tube together with cross sections of the vertical velocity and entropy in the computational volume. The frame on the left is the initial condition while the frame on the right sampled the simulation when $10 \%$ of the flux in the original tube has reached the surface.

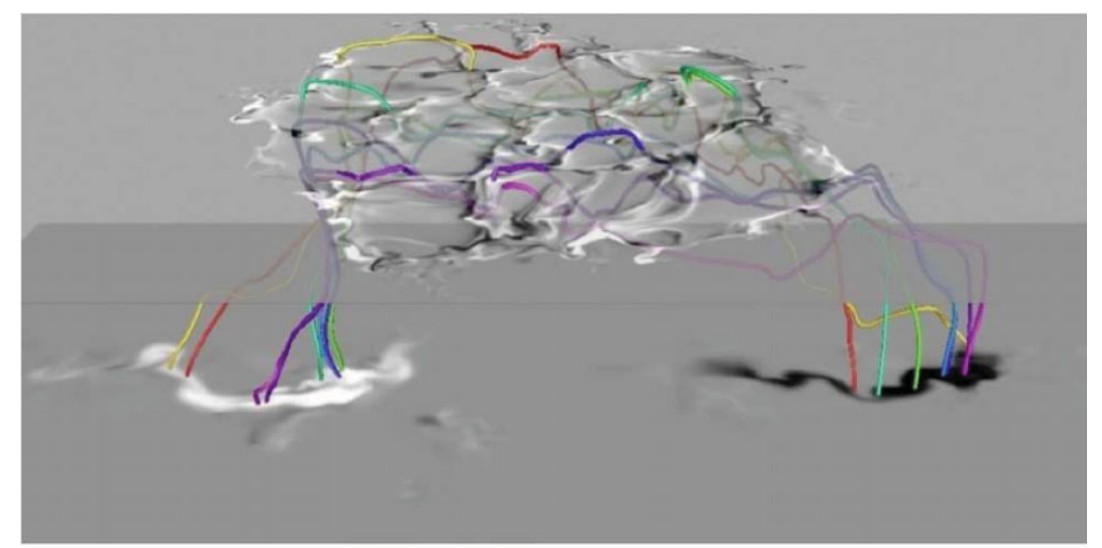

Figure 5. 3D slices of the magnetic flux at $5000 \mathrm{~km}$ and just below the surface together with a few example field lines.

The pond emergence phenomena coupled with the realization that an appreciable fraction of the magnetic field has to be appearing and disappearing on the time scale of tens of minutes seems to argue against feature recognition and tracking schemes as a good methodologies for characterizing the majority of the ubiquitous QS fields that can now be observed at the sub-arc second level. Below some distinguishing characteristics of QS fields are described that are based on characteristics of the flux distributions determined pixel by pixel. 


\section{Data Analysis}

As a first step in trying to develop a diagnostic to characterize the magnetic fields in different solar regions, histograms of the integrated V (IV) signal were created. These histograms are good approximations of the distribution of LOS flux values in the sampled regions. Because of the wide range in the number of points with a given signal, distribution functions were the main focus of the initial analysis. Figure 6 shows an example magnetic map and the histogram of pixel IV signal values. The core of the histogram, the values near zero signal, are well fitted by Gaussians in the example shown as well as all the other maps measured. A lower limit to the amount of area covered by detectable field in the maps can be estimated by subtracting the number of pixels in the Gaussian fit to the core from the total number of pixels in the entire map. In the case illustrated, $24 \%$ of the area of the map have signals outside of the Gaussian core and $71 \%$ of the total unsigned signal results from pixels outside of the Gaussian core.
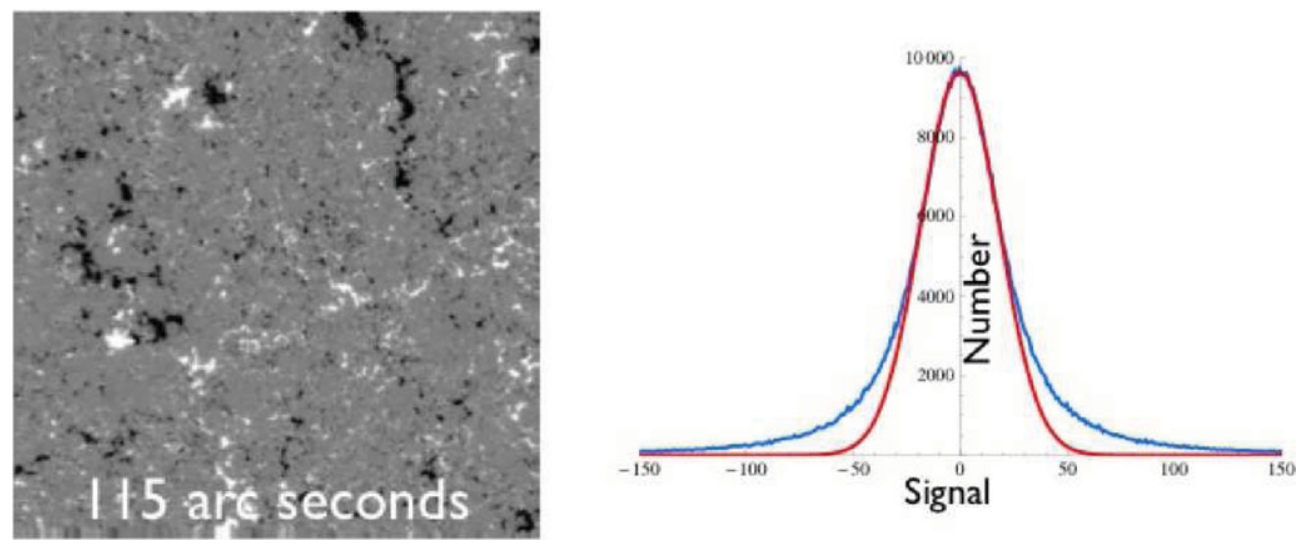

Figure 6. SOT SP (left) on $3 / 05 / 2009$ centered at $(4.1,8.1)$ arc seconds with respect to disk center. The map was $768 \times 768$ with 0.149 arc seconds pixels. The right panel shows in blue the histogram of the magnetic signals in the map. Shown in red is the best fit Gaussian to the core of the histogram. $24 \%$ of the points in the map are outside the Gaussian core and they represent $71 \%$ of the total unsigned signal in the map.

The map shown in figure 6 is roughly $115 \times 115$ arc seconds so should contain several supergranulation cells. It is possible to identify the cell boundaries with a mask that isolates the higher signal regions. By dilating the high flux map and inverting it a map is created that isolates the cell interiors (see figure 7). This map shows an essentially uniform mixture of polarities. On a quick glance it is even difficult to note the blank areas caused by the removal of the network flux.

With the separation of the high and lower values points distribution functions can be created from the entire map as well as the network and cell interiors separately. Figure 8 shows distribution functions for the entire map and those of the high value positive points. The power law indices of the distribution functions are $-2.29,-2.34$, and -2.34 for the negative, positive, and positive high value points, respectively.

Figures 9 through 11 summarize the major results of the set of measurements on magnetic maps in QS over a range of radial positions. Figure 9 shows the variation of histogram slope with radial position. In this figure the magnitude of the indices are shown (all measured indices are negative). A quadratic increase in the slope toward both the polar and equatorial limbs is observed. Figure 10 shows the total (unsigned) and mean 

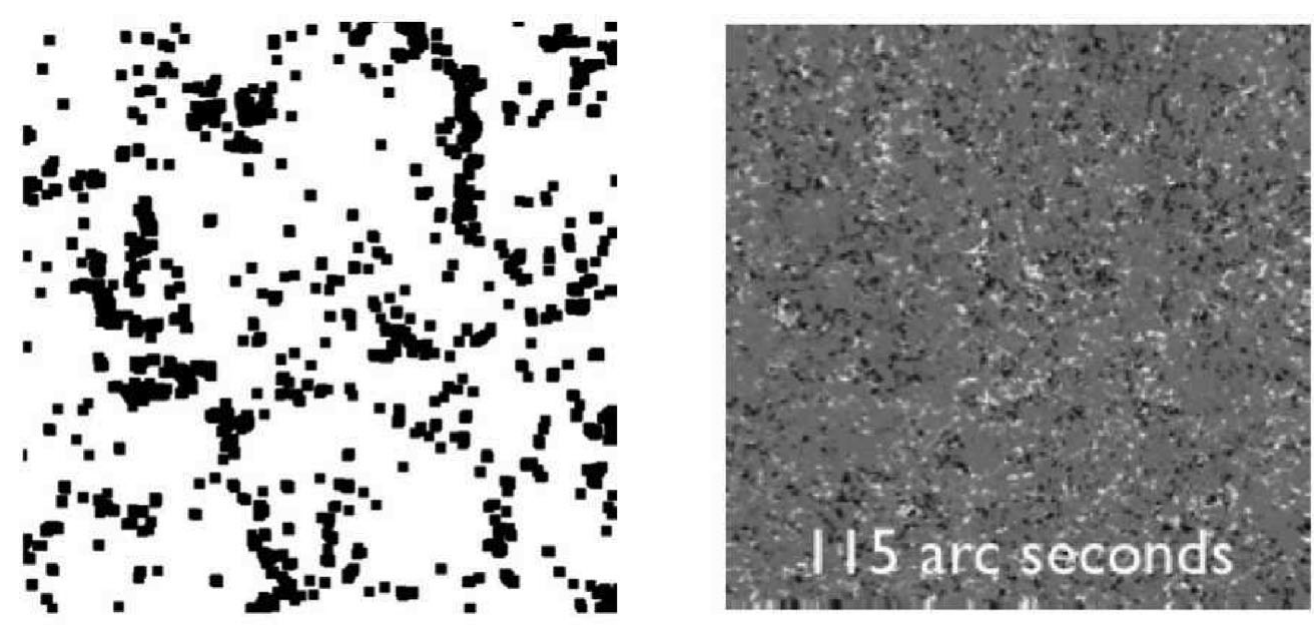

Figure 7. The left hand panel is a mask obtained by dilating and then inverting the high signal map, while the right hand panel is the result of applying the mask to the magnetogram.
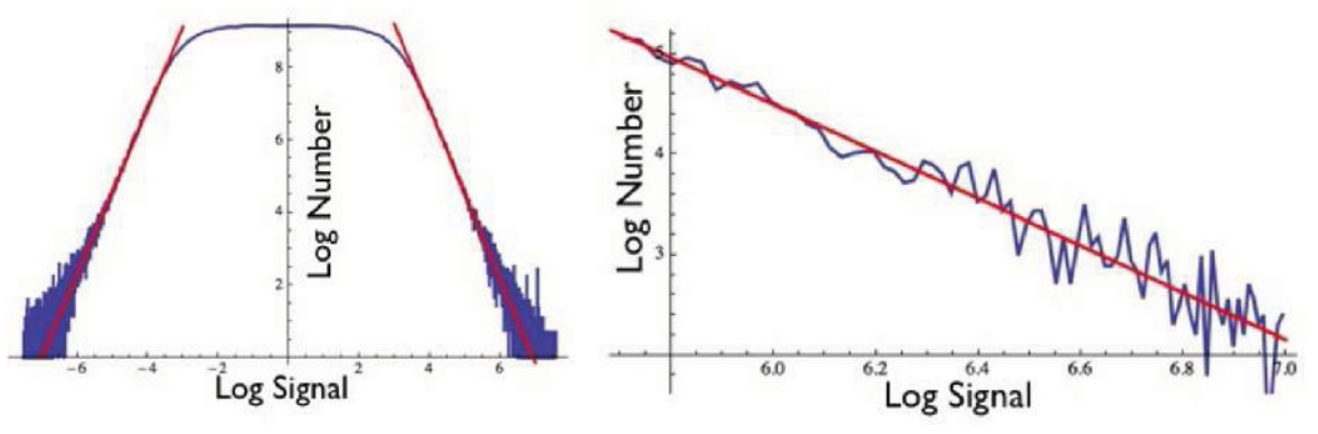

Figure 8. In the left hand panel is the distribution function of the full magnetogram overlaid with the best fit lines to the indices of the positive and negative map points, which are -2.29 and -2.34 for the negative and positive points, respectively. The right hand panel has an expanded scale distribution function of the high value positive points isolated by the high signal mask. Here the index is also -2.34 .

flux as a function of radial position for ONLY those points in the inner network. This figure demonstrates that the average signal in the inner network is near zero for all radial positions. Figure 11 is a similar figure for the value of mean and total flux for high flux points - the network points. Here there is significantly more variation in the value of the mean flux, while the total flux decreases quadratically but only weakly toward the limbs.

\section{Summary of QS Observations}

A notable feature of the distribution functions is that the power law index decreases from -2.3 at disk center to -4 at the limb. If the distribution of the amplitude of individual features was Gaussian such large negative indices would not occur. In order to create the observed power law for an ensemble of magnetic features requires that the peak amplitude of the features has a power law distribution of $-0.5 \pm 0.2$. The drop in 


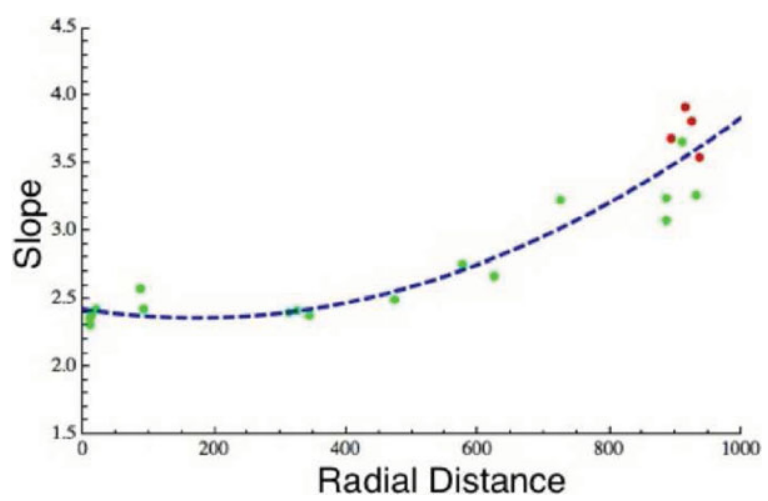

Figure 9. Plot of average slope of distribution functions versus radial position on the disk. The red dots are from equatorial locations. The dashed curve is the best fit to the data to all points. The best fit is of the form $a+c x+b r^{2}$, where a,b,c= $\left(2.4,-7.4 \times 10^{-5}, 2.2 \times 10^{-6}\right)$.

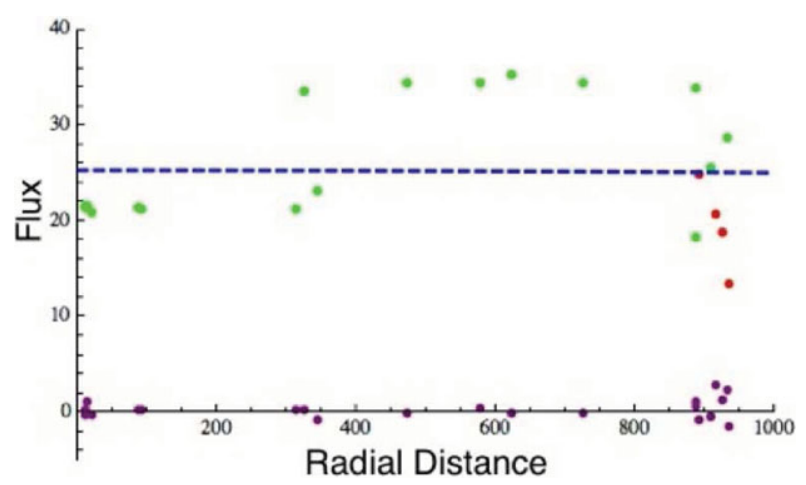

Figure 10. Plots of the unsigned flux in the regions of the quiet sun well outside of the network (green) versus radial position. In red are points near the equator. Shown in purple are the values of the average signal. In all regions the average signal is near zero which shows that in the inner network regions the polarity is well balanced. The ratio average to the flux unsigned flux is 72.6 . The dashed line is the best fit to the unsigned flux data it shows essentially no change in the unsigned flux from disk center to the limbs.

the indices toward the limb indicates that the high amplitude features are more vertical that those of lower amplitude.

More interesting is the observation that the internetwork fields are very well balanced on the scale of the sample boxes that are 115 arc seconds square, or that contain a few supergranules, independent of position on the solar disk. This is true even if the supergranular boundaries that they are internal to are not nearly as well balanced. This property could be caused by local dynamo action or equally likely just a consequence of the stitching cause by the granulation downflows into a rising pond of horizontal flux. 


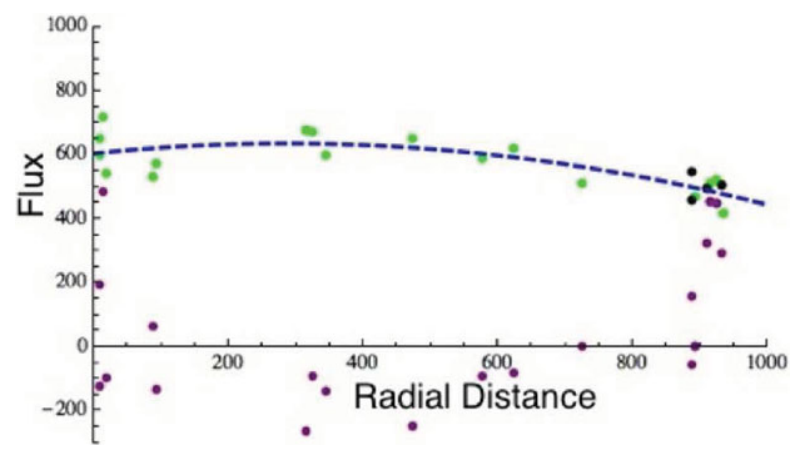

Figure 11. Plots of unsigned flux (green) and of the average flux (purple) versus radial position for network regions. Shown in large black points are total fluxes for the equatorial regions, The dashed curve is a quadratic fit to the unsigned flux data. 